\title{
Transforming the Campus into a digitally augmented learning space using etiquetAR
}

\author{
Mar Pérez-Sanagustín ${ }^{1}$, Javier Melero ${ }^{2}$, Davinia Hernández-Leo ${ }^{2}$, Carlos Delgado \\ Kloos ${ }^{1}$ and Josep Blat ${ }^{2}$ \\ ${ }^{1}$ Dpto. de Ingeniería Telemática, Universidad Carlos III de Madrid, Avd. Universidad 30, \\ 28911 Leganés, Madrid \\ ${ }^{2}$ ICT Department, Universitat Pompeu Fabra, C/Roc Boronat 138, 08018 Barcelona, Spain \\ \{mmpsanag,cdk\}@it.uc3m.es, \{javier.melero, davinia.hernandez\}@upf.edu
}

\begin{abstract}
AR is a web-authoring application designed towards learning purposes to support practitioners in the design of activities based on QR codes. This poster illustrates how etiquetAR $\mathrm{QR}$ codes were used to augment a University Campus as a support for a gamified tag-based learning activity. The QR codes generated change its behavior depending on the profile of the student interacting with the tag. The results show the successful application of etiquetAR in a real educational context.
\end{abstract}

Keywords: Augmented spaces, mobile learning, QR tags, personalization, game, user study

\section{Augmenting the University Campus with etiquetAR}

Tagging technologies such as QR codes offer outstanding possibilities for transforming spaces into digitally augmented learning spaces. Tags, when attached to particular locations, add a digital layer of information that transforms the physical surrounding extending users' learning experience [1]. Although there are tools for generating QR codes, to the best of our knowledge, there are no tools oriented towards learning purposes. etiquetAR ${ }^{1}$ [2] has been designed towards educational purposes with the following functionalities: (1) creating, editing and deleting QR codes with one resource per code, (2) associating profiles to each of these resources and (3) organizing codes into collections. The codes can be accessed using any QR reader installed in the students' smartphone and commented if necessary. Teachers can use their smartphones to delete and even hide an eventual inappropriate comment during the activity, on runtime.

etiquetAR was used to generate $17 \mathrm{QR}$ codes for augmenting the Campus of the University Pompeu Fabra (UPF). Two teachers designed these codes as a support for a learning activity in which first-year undergraduate students registered to the Engineering Bachelor's degrees in Informatics, Telematics and Audiovisual Systems were invited to learn about the campus services and areas. The codes contained three questions, each associated to one profile corresponding to one of the three Bachelor's

1 etiquetAR Website: http://etiquetar.com.es 
Degrees. Since each Bachelor Degree follows a curriculum focused on different areas of ICT, it was especially interesting to show the students distinct information when accessing to the tags. Also, the tags were designed so as to let students to add comments and see comments left by others on-the-fly, since the tags are dynamically updated on runtime with students' contributions. Once designed, the teachers distributed and attached the tags to 17 locations along the Campus areas.

\section{A gamified tag-based learning activity}

The activity was prepared for the first week of the course "Introduction to Information and Communication Technology" (IICT). In this activity, students had to explore the campus looking for the QR codes and solving the different questions contained. A mobile application was implemented specifically for the activity to offer a gamified environment for the students. The mobile application was used to guide the students along the activity flow that required the interaction with the different QR codes. This application also included a QR reader and was in charge of producing log files registering the students' interactions with the tags. The computational representation of the game followed a conceptual model or defining educational mini-games [1].

The results show that etiquetAR was successfully used by the teachers and very well adopted by the students in this educational context. First, the teachers agree that etiquetAR is a usable tool and a good support for designing and enacting tag-based interactive activities. One of the teachers says: "eitquetAR is a useful and simple tool that allows you to create in few steps $Q R$-codes, edit and associate them to different resources and profiles". Second, teachers value the profile functionality and the log files collected indicate that most of students accessed only the information related with their course profile. Third, students agree that the activity helped them to locate themselves in the campus while learning about the different areas and services. Also, teachers indicate that QR codes are especially interesting in "closed controlled spaces where the technology GPS does no work" [Teacher A] and "in situations in which you expect the user to voluntarily access the information in the tag" [Teacher B].

\section{References}

1. Rouillard, J., Laroussi, M.,: PerZoovasive: contextual pervasive QR codes as tool to provide an adaptive learning support, In: 5th Interantional Conference on Soft Computing as transdisciplinar science and Technology, pp. 542-548 (2008)

2. Pérez-Sanagustín, M., Martínez, A. and Delgado Kloos, C.: etiquetAR: tagging learning experiences, EC-TEL 2013, 17-21 September 2013, Paphos, Cyprus (in press). Smith, K.: The future of mobile interpretation. In: Proceedings in Museums and the Web (2009)

3. Melero, J., Hernández-Leo, D., Blat, J.: Towards the Support of Scaffolding in Customizable Puzzle-based Learning Games, In Proceedings of the 11th International Conference on Computational Science and its Applications (ICCSA), Santander, Spain, pp. 254-257 (2011) 